\title{
Revisional Bariatric Surgery: Focus on Quality of Life
}

\author{
Claire Alexandra Zhen Chew ${ }^{1}$, Asim Shabbir ${ }^{2, *}$ \\ ${ }^{1}$ Department of Surgery, National University Hospital; ${ }^{2}$ Department of Surgery, Division of Upper Gastrointestinal Surgery, National University Hospital, Singapore
}

Bariatric surgery is considered to be the most effective treatment for morbid obesity. At present, revisional surgery is considered in patients who experience complications, or in whom the intended weight loss is not achieved. However, as there is no consensus on what constitutes failure of primary surgery, there are no guidelines on who should receive revisional surgery. Physical parameters alone may be insufficient and quality of life has emerged as an alternative to provide a holistic appraisal of the outcome of primary surgery and the need for further revisional surgery in bariatric patients. Quality of life surveys such as short form health survey (SF-36) or Moorehead-Ardelt II (MA-II) assess the patients' perception of their weight and can also be used to assess the impact of post-operative complications such as gastro-esophageal reflux disease or dysphagia. However, unrealistic expectations of weight loss have been shown to be prevalent in bariatric patients and patients who seek revisional surgery on the basis of disappointment with the primary outcome are unlikely to be satisfied with the revisional outcome. Indications for re-operative surgery must be tailored to improve the quality and longevity of each individual patient's life. Long term studies are required to investigate and validate quality of life as an indication for revisional surgery.

Key words: Weight loss, Bariatric surgery, Quality of life

\author{
Received February 26, 2017 \\ Reviewed March 23, 2017 \\ Accepted April 20, 2017 \\ ${ }^{*}$ Corresponding author \\ Asim Shabbir \\ http://orcid.org/0000-0002-2490-6478 \\ Department of Surgery, Division of \\ Upper Gastrointestinal Surgery, National \\ University Hospital, NUHS Tower Block, \\ Level 8, 1E Kent Ridge Road, Singapore \\ 119228, Singapore \\ Tel: $+65-6772-9200$ \\ Fax: +65-6777-8427 \\ E-mail: cfsasim@nus.edu.sg
}

\section{INTRODUCTION}

Morbid obesity is a major public healthcare burden. Bariatric surgery is now widely accepted as the most effective method in the treatment of morbid obesity and its related co-morbidities, such as type 2 diabetes mellitus. ${ }^{1,2}$ Although rarely associated with mortality, bariatric surgery is considered unsuccessful when the intended weight loss is not achieved or when complications arise, thereby necessitating further revisional surgery. The percentage of revisional bariatric procedures varies across different healthcare systems, ranging from as low as $2 \%$ in the UK and Russia to up to $17-20 \%$ in Sweden. ${ }^{3,4}$ Unfortunately, these statistics cannot be meaningfully compared without acknowledging that there is a lack of standardized guidelines on the indications for revisional surgery after primary bariatric procedures.

\section{What is revisional surgery?}

As in other surgical specialities, revisional procedures in bariatric surgery are performed in patients who have an inadequate response or no response to initial therapy, as well as in those who have complications from therapy and require reversal or correction of these complications. Table 1 shows the nomenclature used by the American Society for Metabolic and Bariatric Surgery Revision Task Force to define revisional bariatric procedures. ${ }^{5}$ However, the problem in the current approach to revisional bariatric surgery lies in that there is no agreement on what constitutes failure of initial therapy.

\section{Current indications for revisional surgery}

A systematic review looking at the definition of failure of primary bariatric surgery concluded that there was inconsistent reporting of the reasons for failure and that a predominant number of studies concerning revisional operations failed to report their selection cri- 
Table 1. Types of revisional bariatric surgery

\begin{tabular}{llc}
\hline Type of operation & \multicolumn{1}{c}{ Indication } & Example \\
\hline Conversion & $\begin{array}{c}\text { Procedures that change an index procedure to a different } \\
\text { type of procedure }\end{array}$ & $\begin{array}{c}\text { Laparoscopic adjustable gastric banding to RYGB, laparoscopic sleeve } \\
\text { gastrectomy or duodenal switch }\end{array}$ \\
Corrective & $\begin{array}{c}\text { Procedures that address complications or inadequate response } \\
\text { of a previous bariatric operation }\end{array}$ & $\begin{array}{c}\text { Endoscopic therapy to reduce the pouch and gastrojejunal stomal size after RYGB } \\
\text { Reversal }\end{array}$ \\
Procedures that restore original anatomy & $\begin{array}{c}\text { Reversal of RYGB for severe complications such as intractable nausea, vomiting, } \\
\text { psychological issues, excessive weight loss }\end{array}$ \\
\hline
\end{tabular}

RYGB, Roux-en-Y gastric bypass.

teria. ${ }^{6}$ They noted that amongst the studies reviewed, the most frequently used definition for failure of the primary procedure was $<50 \%$ of excess weight lost (EWL), with or without a body mass index (BMI) of greater than $35 \mathrm{~kg} / \mathrm{m}^{2}$ at 18 months post-operation. Whilst weight loss is a straightforward and easily applied method to select patients for revisional surgery, there are some pitfalls in this approach. \%EWL was originally proposed by Reinhold ${ }^{7}$ in 1982 as an outcome measure of a patient's response to bariatric surgery. During that time, the indication for surgical treatment was being $100 \%$ above one's ideal weight, and an increase in mortality was associated with patients who had more than $50 \%$ excess weight. ${ }^{8}$ It is now well recognized that increased morbidity and mortality are associated with even lower excess weights. This cut-off of 50\% EWL is thus a reinterpretation of the principle proposed by Reinhold and is in actuality a rather arbitrary target of success. \%EWL as it is used today, describes weight loss relative to an ideal BMI of 25, which is also problematic because this is not a feasible target for patients who are $>50 \mathrm{~kg} / \mathrm{m}^{2} .{ }^{9}$ Furthermore, recently published studies have found other outcomes measures such as expected BMI loss (when corrected for pre-operative BMI $)^{9}$ or percentage weight change, as is used in major prospective studies, to be more accurate methods of weight loss reporting. ${ }^{10,11}$

However, looking more broadly, there is also an observable variation in the expected weight loss depending on the operation performed. A recent meta-analysis found that there was a difference in mean reduction in BMI between different procedures: $12-17 \mathrm{~kg} /$ $\mathrm{m}^{2}$ for sleeve gastrectomy compared to only $2 \mathrm{~kg} / \mathrm{m}^{2}$ in adjustable gastric banding showing that if weight loss outcomes were to be used as an indication for revision, then it would need to be adjusted for the primary bariatric procedure itself as well. ${ }^{12}$ Various studies have also reported that $20-30 \%$ of patients fail to achieve successful weight loss ${ }^{13,14}$, the implication being that these patients should then require revisional surgery. Unfortunately, using weight loss alone as an indication does not take into account patients who may be 'metabolically healthy obese' i.e. patients that may fail to achieve target weight loss but do not suffer from metabolic complications associated with morbid obesity. ${ }^{15}$ Revisional surgery is associated with a higher risk of complications than primary bariatric surgery, and in these patients this risk is unlikely to outweigh the benefits of further weight loss that may be achieved with re-operation. It also fails to account for patients who achieve the targeted weight loss but may still be at risk of obesity related co-morbidities as it has been shown that reduction in \%EWL does not necessarily correlate with an unequivocal reduction in health risk. ${ }^{16}$ It is interesting to note that there were no studies that considered non-resolution of co-morbidities as an indication for revisional surgery. ${ }^{6}$ These concerns highlight a need for more selective criteria in determining candidates for revisional surgery such that patients who would benefit from re-operation are able to receive it.

\section{Quality of life as an indicator}

To redefine the selection process for revisional surgery, it may be worth reflecting on the definition of revisional surgery itself. As laid out by the American Society of Metabolic and Bariatric Diseases task force, revisional surgery can be broadly categorised into conversions, corrective procedures or reversals to tackle inadequate response to surgery, complications or intolerance to complications brought on by surgery. ${ }^{5}$ From a clinician's perspective, inadequate response to surgery is measured by weight loss reported or remission of co-morbidities, however it may be valuable to also consider the patient's perspective. It is known that often patients seek bariatric surgery on the basis of severity of psychological distress associated with their morbid obesity, rather than absolute weight gain above the norm. ${ }^{17}$ Hence, their opinion of the success or failure of 
treatment is likely dependent on whether their expectations of the outcomes of surgery have been met. This can be measured using quality of life surveys such as short form health survey (SF-36) or Moorehead-Ardelt II (MA-II), which both take into account factors such as appearance, physical health, social functioning, mental health and so on. These surveys have both been used in studies looking at quality of life after bariatric procedures. ${ }^{18}$ Patients that feel that the improvement in their quality of life is not as much as expected may feel that the primary bariatric procedure was ineffective. A longitudinal population-based study with surveys conducted at 5 year intervals reported a bi-directional relationship between health-related quality of life and obesity, showing that not only does obesity lead to reduced quality of life, but that impaired quality of life is a predictor of weight gain. The attainment and maintenance of good quality of life should be considered as one of the goals of bariatric surgery, and patients who fail to do so or perform persistently low on quality of life surveys post-operatively can be singled out as candidates that may benefit from revisional surgery. A study conducted by Mohos et al. ${ }^{18}$ compared the quality of life of patients after primary and revisional laparoscopic Roux-en-Y gastric bypass (LRYGB) using both SF-36 and MA-II as measurements of quality of life. Although there was no statistically significant difference found between quality of life in both groups using either survey, the scores achieved by both groups corresponded to acceptable standards of quality of life as compared to the normal European population. More studies like these are needed to investigate the impact of revisional surgery on quality of life in patients.

Complications or intolerance effects from the primary procedures are also strong indications for revisional surgery. In the same study by Mohos et al. ${ }^{18}$, they reported 657 patients who underwent LRYGB and 81 patients who underwent revisional LRYGB of which 62 were for inadequate weight loss, 11 were for gastroesophageal reflux disease (GERD) and 8 were for dysphagia. Of the patients that underwent revision for GERD and dysphagia, all of them had presented post-operatively complaining of symptoms that were affecting their quality of life. In another study, severe nutritional deficiency was noted in $1 \%$ of patients post LRYGB who presented with severe diarrhoea and swelling resistant to pancreatic enzyme replacement and anti-diarrheal agents. ${ }^{19}$ Similarly, another study considered patients that presented almost 5 years post LRYGB with gastroscopy proven bile reflux which manifested as pain, vomiting and dysphagia, revisional surgery was performed in which the alimentary limb was lengthened and the symptoms resolved. ${ }^{20}$ The symptoms that these patients present with are a reflection of the underlying technical complications of the primary procedure that are amenable to correction. Such factors can be examined post-operatively and monitored in addition to weight loss and psychosocial wellbeing, to provide a holistic appraisal of the outcome of primary surgery and assess whether there is a need for a revisional procedure.

However, it is important to recognise that there may be certain pitfalls to using quality of life alone as an indicator for revisional surgery. Obese patients that seek surgical treatment have been shown to have more severe general psychopathology compared to obese patients that choose medical or conservative therapies. ${ }^{17}$ Furthermore, unrealistic expectations of weight loss have been shown to be prevalent in obesity surgery patients. A study of 284 prospective candidates for bariatric surgery, patients stated that their "dream" weight loss was 89+/-8\% EWL, and would be "disappointed" if their weight loss was $49+/-14 \%{ }^{21}$ This is concerning when $50 \%$ EWL is considered by most centres to be successful weight loss. Another study evaluating willingness to accept risk in patients seeking bariatric surgery found that majority of patients interviewed were willing to accept some level of mortality risk to undergo surgery, with $19.5 \%$ of patients willing to accept a risk of at least $10 \% .{ }^{22}$ Given that the weight loss outcomes of revisional surgery are understandably significantly less than that of primary bariatric surgery $^{18}$, patients who seek revisional surgery on the basis of disappointment with the primary outcome are unlikely to be satisfied with the revisional outcome.

It seems that evaluation of quality of life in the context of bariatric surgery needs to performed in conjunction with a psychosocial evaluation of the patient themselves as well. In this way, both the clinician and patient perspectives are accounted for with the added benefit of identifying patients who may have unrealistic expectations or unhealthy cognitive processes, thereby allowing for appropriate interventions to be made. Pre-operative counselling for revisional surgery should be a key step in the process of patient selection, as patient attitudes and expectations will invariably influence the post-operative course. 


\section{CONCLUSION}

Arbitrary indications based alone on BMI or weight should not be used. Indications for re-operative surgery must be tailored to improve the quality and longevity of each individual patient's life. Quality of life after primary bariatric surgery is influenced by the effectiveness of weight loss, any complications sustained and by the patient's perception of the experience. It is valuable to consider it as part of a holistic appraisal of success or failure of the primary procedure, to subsequently decide whether revisional surgery is indicated in the patient. Long term studies are required to investigate and validate quality of life as an indication for revision surgery.

\section{CONFLICTS OF INTEREST}

The authors declare no conflict of interest.

\section{REFERENCES}

1. Gloy VL, Briel M, Bhatt DL, Kashyap SR, Schauer PR, Mingrone $\mathrm{G}$, et al. Bariatric surgery versus non-surgical treatment for obesity: a systematic review and meta-analysis of randomised controlled trials. BMJ 2013;347:f5934.

2. Courcoulas AP, Yanovski SZ, Bonds D, Eggerman TL, Horlick M, Staten MA, et al. Long-term outcomes of bariatric surgery: a national institutes of health symposium. JAMA Surg 2014; 149:1323-9.

3. Kellogg TA. Revisional bariatric surgery. Surg Clin North Am 2011;91:1353-71.

4. Elder KA, Wolfe BM. Bariatric surgery: a review of procedures and outcomes. Gastroenterology 2007;132:2253-71.

5. Brethauer SA, Kothari S, Sudan R, Williams B, English WJ, Brengman M, et al. Systematic review on reoperative bariatric surgery: American Society for Metabolic and Bariatric Surgery Revision Task Force. Surg Obes Relat Dis 2014;10:952-72.

6. Mann JP, Jakes AD, Hayden JD, Barth JH. Systematic review of definitions of failure in revisional bariatric surgery. Obes Surg 2015;25:571-4.

7. Reinhold RB. Critical analysis of long term weight loss following gastric bypass. Surg Gynecol Obstet 1982;155:385-94.
8. Seltzer CC. Some re-evaluations of the build and blood pressure study, 1959 as related to ponderal index, somatotype and mortality. N Engl J Med 1966;274:254-9.

9. Baltasar A, Perez N, Serra C, Bou R, Bengochea M, Borrás F. Weight loss reporting: predicted body mass index after bariatric surgery. Obes Surg 2011;21:367-72.

10. Hatoum IJ, Kaplan LM. Advantages of percent weight loss as a method of reporting weight loss after Roux-en-Y gastric bypass. Obesity (Silver Spring) 2013;21:1519-25.

11. Courcoulas AP, Christian NJ, Belle SH, Berk PD, Flum DR, Garcia L, et al. Weight change and health outcomes at 3 years after bariatric surgery among individuals with severe obesity. JAMA 2013;310:2416-25.

12. Chang SH, Stoll CR, Song J, Varela JE, Eagon CJ, Colditz GA. The effectiveness and risks of bariatric surgery: an updated systematic review and meta-analysis, 2003-2012. JAMA Surg 2014;149:275-87.

13. Christou NV, Look D, Maclean LD. Weight gain after shortand long-limb gastric bypass in patients followed for longer than 10 years. Ann Surg 2006;244:734-40.

14. Sjöström L, Lindroos AK, Peltonen M, Torgerson J, Bouchard C, Carlsson B, et al. Lifestyle, diabetes, and cardiovascular risk factors 10 years after bariatric surgery. N Engl J Med 2004; 351:2683-93.

15. Muñoz-Garach A, Cornejo-Pareja I, Tinahones FJ. Does metabolically healthy obesity exist? Nutrients 2016;8:320.

16. Higa KD, Boone KB, Ho T, Davies OG. Laparoscopic Rouxen-Y gastric bypass for morbid obesity: technique and preliminary results of our first 400 patients. Arch Surg 2000;135: 1029-33.

17. Castellini G, Godini L, Amedei SG, Galli V, Alpigiano G, Mugnaini E, et al. Psychopathological similarities and differences between obese patients seeking surgical and non-surgical overweight treatments. Eat Weight Disord 2014;19:95-102.

18. Mohos E, Schmaldienst E, Prager M. Quality of life parameters, weight change and improvement of co-morbidities after laparoscopic Roux Y gastric bypass and laparoscopic gastric sleeve resection--comparative study. Obes Surg 2011;21:288-94.

19. Biertho L, Biron S, Hould FS, Lebel S, Marceau S, Marceau P. Is biliopancreatic diversion with duodenal switch indicated for 
patients with body mass index $<50 \mathrm{~kg} / \mathrm{m}^{2}$ ? Surg Obes Relat Dis 2010;6:508-14.

20. Swartz DE, Mobley E, Felix EL. Bile reflux after Roux-en-Y gastric bypass: an unrecognized cause of postoperative pain. Surg Obes Relat Dis 2009;5:27-30.

21. Kaly P, Orellana S, Torrella T, Takagishi C, Saff-Koche L, Murr
MM. Unrealistic weight loss expectations in candidates for bariatric surgery. Surg Obes Relat Dis 2008;4:6-10.

22. Wee CC, Hamel MB, Apovian CM, Blackburn GL, BolcicJankovic D, Colten ME, et al. Expectations for weight loss and willingness to accept risk among patients seeking weight loss surgery. JAMA Surg 2013;148:264-71. 